\title{
Analysis of Fracturing Ability Factors of Tight Reservoir
}

\author{
Zhenhua $\mathrm{Li}^{1 *}, \mathrm{Ke} \mathrm{Gail}^{2}$ and Feng Cao ${ }^{1}$ \\ ${ }^{1}$ School of Energy Engineering, Longdong University, Qingyang, China \\ ${ }^{2} \mathrm{~S} c h o o l$ of Chemical Engineering, Longdong University, Qingyang, China
}

\begin{abstract}
Fracturing ability is the characteristic used to measure whether the tight reservoir could be effectively fractured and form network in the process of hydraulic fracturing in order to improve the production of oil and gas. Research shows that the more brittle the reservoir is, the weaker the fracture toughness is, the more developed the natural fracture is, the higher the maturity of organic matter is, the lower the organic carbon content is, and the better the fracturing ability is. In addition, factors such as crustal stress, sedimentary environment and internal structure of the reservoir also affect the fracturing ability. The analysis of reservoir fracturing ability plays a key role in the strata selection, multi-stage fracturing design and prediction of oilfield economic benefits.
\end{abstract}

\section{Introduction}

With the depletion of conventional oil and gas resources, unconventional oil and gas resources, such as shale gas, coalbed gas and tight sandstone gas, have attracted more and more attention[1]. Unconventional oil and gas reservoir generally show the characteristics of tight lithology, low porosity and permeability, and complex micro pore structure. In order to improve the single well production, large-scale fracturing is generally required. The staged fracturing technology of horizontal well promotes the effective development of unconventional oil and gas, but not all strata can achieve ideal production capacity through fracturing, only those strata with high brittleness can form complex fractures[2]. Therefore, the fracturing analysis will play a key role in the fracturing strata selection, multi-stage fracturing design and prediction of economic benefits. In this paper, the factors such as brittleness, fracture toughness, natural fracture and maturity of organic matter that could influence the fracturing ability of reservoirs are discussed.

\section{Connotation of fracturing}

It is generally believed that fracturing ability is the characteristic used to measure whether the tight reservoir could be effectively fractured and form fracture network in order to increase production in the process of hydraulic fracturing[3]. Because fracturing ability is a new concept, there is no unified qualitative evaluation criterion at home and abroad. Chong et al. summarized the successful fracturing methods in North America shale block in the past 20 years, and believed that the fracturing ability is the property that the shale reservoir can be effectively fractured to increase production[4].
Breyer believes that the fracture ability is related to the brittleness and toughness of materials, which can be revealed by elastic modulus and Poisson's ratio. In addition, it can also be reflected by compressive strength and internal friction angle.

The research shows that the fracturing result is not only related to the geological characteristics of reservoir, but also depends on the fracturing technology used. But the fracturing ability reflects the comprehensive characteristics of reservoir in the process of hydraulic fracturing, has nothing to do with the fracturing technology[5]. It can be seen that fracturing ability can be described as the ability to form a complex fracture network in the reservoir and obtain a large enough reservoir reconstruction volume to achieve high economic benefits under the same technology.

\section{Influence factors of fracturing ability}

\subsection{Brittleness}

Reservoir brittleness is the most important factor affecting the fracturing ability. For oil and gas reservoirs, brittleness is generally defined as the difficulty of transient change before rock fracture, which indirectly reflects the complexity of fracture, and can be quantitatively characterized by brittleness index[6]. Brittleness index is an important indication for selecting high-quality unconventional oil and gas reservoir, which can significantly affect the stability of well wall, and is the key to evaluate the mechanical properties. Generally speaking, the higher the brittleness index is, the harder the formation is, and the better the fracturing ability is, and then it can rapidly form complex network fractures, which is conducive to oil and gas development; on the contrary, the reservoir with low brittleness index only 
forms simple fractures, which is not favorable for oil and gas seepage.

The elastic modulus and Poisson's ratio are the main mechanics parameters to indicate the reservoir brittleness. The elastic modulus reflects the ability to maintain the fracture, and Poisson's ratio reflects the ability to fracture under pressure[7,8].The higher the elastic modulus is, the lower Poisson's ratio is and the greater the brittleness index is(Fig. 1, 2).

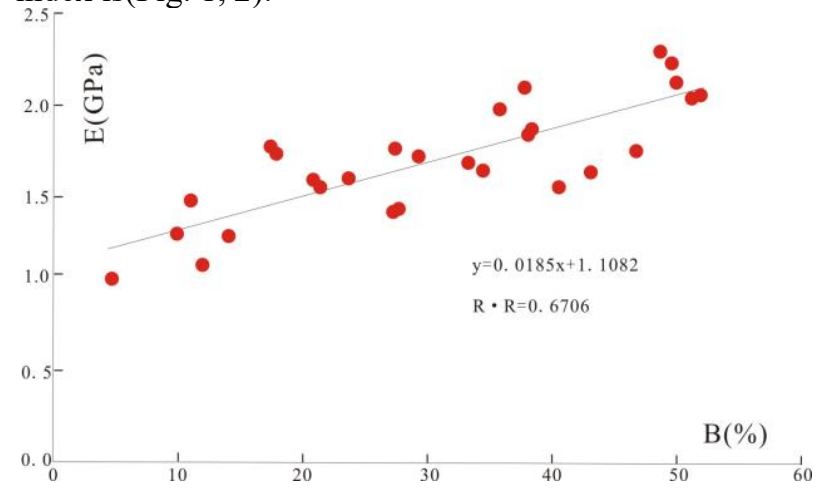

Fig. 1 Relationship between elastic modulus and brittleness index.

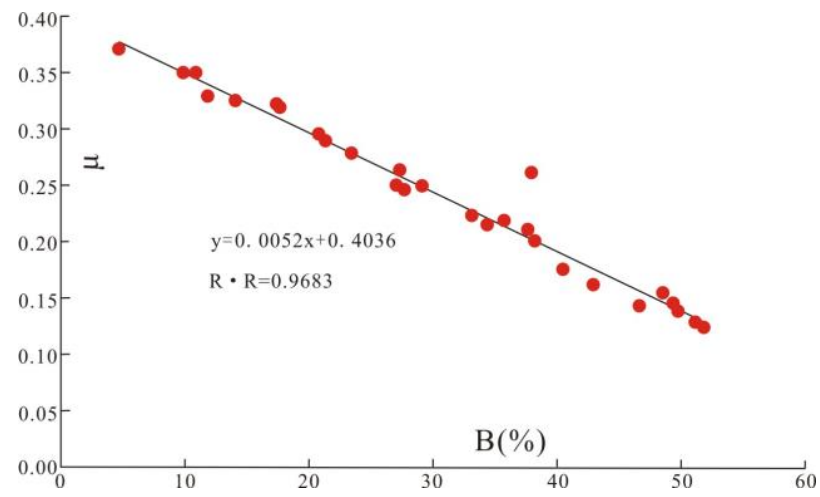

Fig. 2 Relationship between Poisson's ratio and brittleness index.

The elastic modulus of formation ranges from $10 \mathrm{GPa}$ to $80 \mathrm{GPa}$, and Poisson's ratio is from 0.15 to 0.4 . In a long geological period, natural fractures are developed in the formation with higher brittleness index under the influence of structural movement, which is sensitive to the fracturing operation and can form complex network rapidly. While the formation with low brittleness index is easy to form simple double wing fracture.

\subsection{Fracture toughness}

Fracture toughness, also known as the critical stress intensity factor, is a key parameter in fracture analysis. It is a basic property of material and generally independent of the geometry and load of the fractures[9]. In petroleum geology, fracture toughness can indicate the difficulty degree of reservoir fracturing, and reflect the ability of maintaining the fracture to extend forward. The toughness index can also be represented by the normalized toughness. The smaller the fracture toughness index is, the higher the fracturing ability is (Fig. 3).

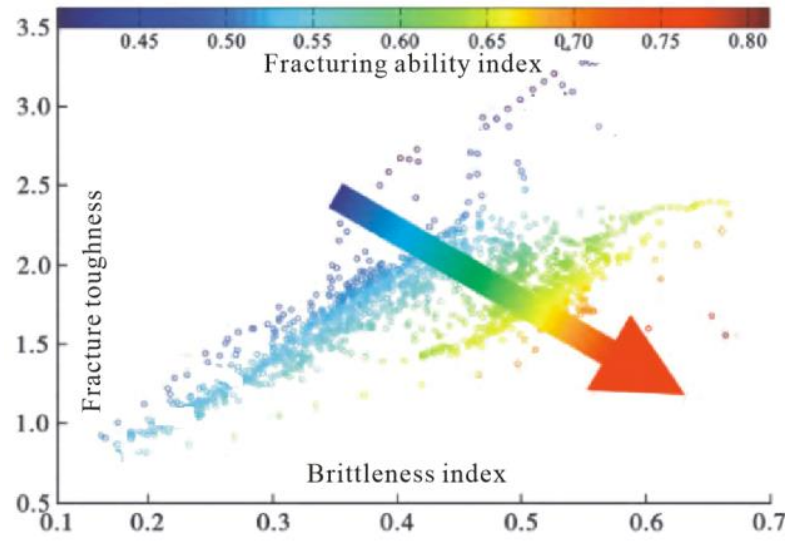

Fig. 3 Relationship between fracturing ability, brittleness index and fracture toughness of tight reservoir.

\subsection{Natural fractures}

Generally speaking, the rock is under the influence of three mutually vertical and unequal principal stresses, one is vertical and the other two are horizontal. In the process of structural movement or hydraulic fracturing, when the vertical principal stress is less than the other two horizontal stresses, it is easy to form horizontal fractures in the rock, and when the vertical principal stress is greater than others, it is easy to form vertical fractures. The existence of natural fractures is the manifestation of uneven crustal stress, which means the formation stress is weak there. The existence of natural fractures reduces the tensile strength of rock, and changes the crustal stress near the wellbore, which has an impact on the formation and extension of induced fractures[10]. Therefore, the more developed the natural fractures are, the higher the fracturing ability is.

Natural fractures can enhance the effect of fracturing operation, and compared with the formation without natural fractures, this kind of formation just need as low as $50 \%$ pressure in the process of fracturing. The natural and induced fractures interact with each other. The fracturing fluid flows into the reservoir through the natural fracture in order to generate induced ones. The induced fractures can also cause the opening of the natural ones to let more fracturing fluid to enter. In the reservoir, natural and induced fractures together can improve oil and gas production.

\subsection{Diagenesis}

In different diagenesis stages, the mineral form, clay mineral composition and pore type are different. From the perspective of the whole process of hydrocarbon generation of organic matter, shale brittleness is mainly affected by the composition of clay minerals at the stage of low maturity. With the increase of maturity, the brittleness of shale minerals improves, and at the same time, due to the increase of hydrocarbon generation and expulsion, the porosity of reservoir becomes greater, and the fracture is more developed, so the fracturing ability is improved. The higher the maturity, the faster the fracturing ability is improved (Figure 4). 


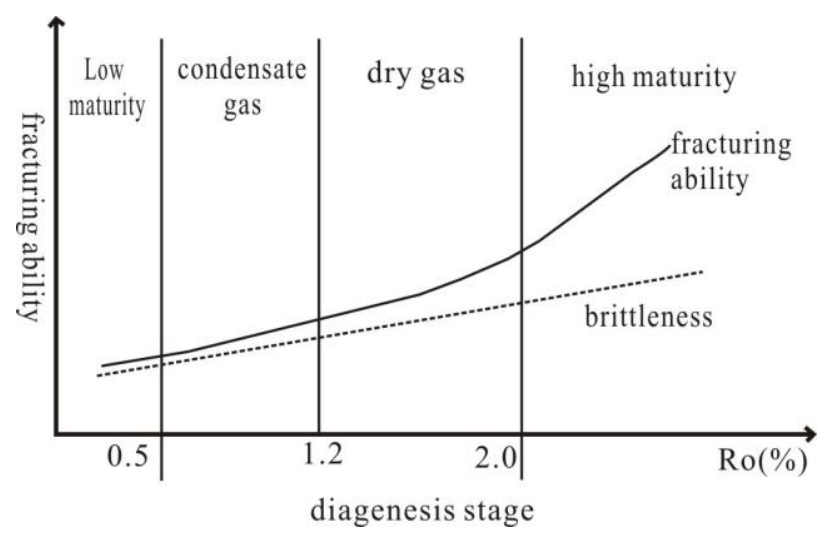

Fig. 4 Relationship between fracturing ability and diagenesis stage

\subsection{Organic carbon content}

The statistical data of organic carbon content and rock elastic parameters in the reservoir show that the organic matter will also affect its brittleness by reducing the elastic parameters. Generally, the higher the organic matter content is, the stronger the plasticity of the reservoir is, the lower the brittleness is, and the more unfavorable for fracturing (Fig. 5).

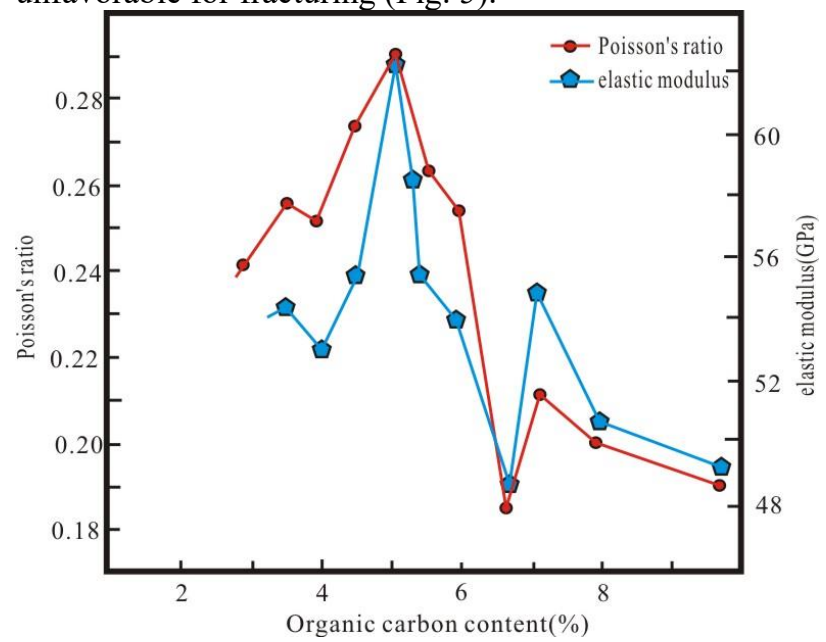

Fig. 5 Effect of organic carbon content on reservoir elastic parameters

\section{Conclusion}

Fracturing ability is the characteristic used to measure whether the tight reservoir could be effectively fractured and form network in order to increase production in the process of hydraulic fracturing. It reflects the comprehensive characteristics of reservoir and has nothing to do with fracturing technology. Brittleness is the most important factor that affects the fracturing ability of reservoir. The greater the brittleness index is, the better the fracturing ability is. The fracture toughness reflects the ability of maintaining the fracture to extend forward. The weaker the fracture toughness is, the higher the fracturing ability is. At the same time, natural fractures and the maturity of organic matter also affects it. The more developed the natural fractures, the higher the maturity, the better it is. The analysis of fracturing ability plays a key role in the strata selection, multi-stage fracturing design and prediction of oilfield economic benefits.

\section{Acknowledgments}

This work was financially supported by Xifeng Technology Bureau Fund Project, project number XK2019-10 , and by Department of industry and information technology Funds of Gansu Province, project number GGLD-2019-066.

\section{References}

1. JinLan, Guiwen Wang, Zhuiying Fan. (2016) Research progress on the evaluation methods of brittleness index of unconventional oil and gas reservoir. Petroleum Science Bulletin. pp. 330-341.

2. Chenchen Zhang, Dazhong Dong, Yuman Wang. (2017) Research progress of shale reservoir brittleness. Xinjiang Petroleum Geology. pp. 111-117.

3. Junliang Yuan, Jingen Deng, Dingyu Zhang. (2014) Fracturing evaluation technology of shale gas reservoir. Petroleum Journal.(34): 523-527.

4. Chong K.K., Griese R.W., Jaripatke,O.A. (2010) A Completions guide book to shale-play development: a review of successful approaches toward shale-play stimulation in the last two decades. China: SPE International Oil and Gas Conference and Exhibition. Beijing.

5. Jianmeng Sun, Zhilei Han, Ruibao Qin.(2015)Well logging evaluation methods for fracturing ability of tight gas reservoir. Petroleum Journal.(36): 74-80.

6. Haiyan Diao. (2013)Mechanical properties and brittleness evaluation of shale reservoir. Journal of rock.(9): 3300-3306.

7. Qinghui Li,Mian Chen,Yan Jin.(2012)Laboratory evaluation methods and improvement of shale brittleness. Journal of rock mechanics and Engineering.(31);1680-1685.

8. Quan Gao, Junliang Tao. (2015) Laboratory study on the mechanical behaviors of an anisotropic shale rock. Journal of Rock Mechanics and Geotechnical Engineering.(7):213-219.

9. Mian Chen, Yan Jin, Guangqing Zhang. (2011) Rock mechanics foundation of Petroleum Engineering. Petroleum Industry Press. Beijing. pp.38-41.

10. Jinzhou Zhao, Hai Yang, Yongmin Li. (2014) Stability analysis of natural fractures in the process of hydraulic fracturing. Natural Gas Geoscience. (25):402-408. 